\title{
Uso de las plantas nativas por una comunidad de cabreros de Las Vegas de la quebrada de Tulahuén, Región de Coquimbo, Chile
}

\author{
Usage of native plants by a community of herdsmen at the meadows of the ravines of \\ Tulahuén, Region of Coquimbo (Chile)
}

\author{
Eduardo Jaime Muñoz ${ }^{*} \&$ Rodrigo Villaseñor Castro ${ }^{2}$
}

\section{RESUMEN}

Se presentan los usos de las plantas vasculares existentes en Las Vegas de la quebrada de Tulahuén, comuna de Monte Patria, Región de Coquimbo, utilizadas por una comunidad de cabreros en un ecosistema de estepa alto andina precordillerana. A las personas que residen en el sector se les aplicaron entrevistas. Continuando con el trabajo en terreno para estudiar las poblaciones vegetales se hicieron 10 parcelas de 50 x $50 \mathrm{~m}$.

En su gran mayoría los nombres comunes de las especies fueron reconocidas por los cabreros, de un total de 93 plantas entre nativas y endémicas. Varias de ellas tienen más de un uso: 32 especies son utilizadas como combustible, 72 como forrajeras, 35 como medicinales y 13 para hacer artesanía. A manera de conclusión este estudio deja registrados los usos de las plantas nativas y las comunidades vegetales de la zona. Los resultados de la investigación serán un aporte al conocimiento etnobotánico a través de un registro de los usos que les dan los cabreros a las diferentes plantas nativas que crecen en esta zona precordillerana.

Palabras clave: estepa alto andina, etnobotánica, trashumancia

\begin{abstract}
Presenting the uses of existing vascular plants that occur in the meadows of the ravine of Tulahuén, commune of Monte Patria, Coquimbo Region, used by a community of goat herders in a steppe ecosystem of high Andean foothills. People who reside in the area were interviewed. 10 plots of $50 \times 50$ meters were made to study the plant populations; In the vast majority the common names of species were recognized by the goat herders, a total of 93 native plants between native and endemic, several of them have more than one use: 32 species are used as combustible, 72 as fodder, 35 as medicinal and 13 are used to make handicrafts. This work leaves registered the uses of native plants by goat herders; and plant communities, found in the study area.

The research results will be a contribution to ethnobotanical knowledge through a record of uses that the goat herders give to various native plants growing in these Andean foothills.
\end{abstract}

Keywords: high Andean steppe, ethnobotany, trashumance.

\section{Introducción}

La comunidad de cabreros estudiada en este trabajo se ubica en la localidad de Las Vegas de la quebrada de Tulahuencito. El lugar pertenece a la comuna de Monte Patria, provincia del Limarí, Región de Coquimbo. Para elaborar este estudio se trabajó con tres majadas con un total de 2.100 cabras: la majada de los Morosos compuesta por 10 personas con 800 cabras, los Villalobos compuesta por 8 personas con 1.000 cabras y los Tapias compuesta por 5 personas con 300 cabras. El paisaje natural dentro del área estudiada forma parte de la estepa alto andina, formación vegetal distribuida desde la cordillera de los Andes árida y semiárida, extendiéndose desde el extremo norte, en el límite con Perú y Bolivia, hasta las montañas andinas de la Región del Maule (Gajardo 1994).

1 Universidad Nacional de San Juan, Ciudad de San Juan. Argentina.

2 Laboratorio de Botánica, Facultad de Ciencias, Universidad de Playa Ancha de Valparaíso.

* Autor por correspondencia: rvillac@upla.cl

Fecha de Recepción: 11 noviembre, 2017.

Fecha de Aceptación: 21 mayo, 2018.

DOI: http://dx.doi.org/10.4067/S0718-34292018005000201. Publicado en línea: 20-julio-2018. 
En este ambiente de estepas alto andinas existe una comunidad de personas dedicadas a la actividad caprina, que se desenvuelve cercana al poblado de El Tayán y Tulahuén. Los cabreros suben sus cabras a la cordillera los meses de octubre y noviembre, arreando su rebaño con sus familias. Los niños se quedan en la escuela y sólo van personas mayores, debido a lo inhóspito del clima cordillerano. Los cabreros utilizan las plantas nativas de su entorno natural con fines combustibles, forrajeros, medicinales y artesanales. Por esta razón, la flora nativa es un recurso indispensable para el desarrollo de la actividad ganadera en esta comunidad. La trashumancia -con veranada y sin veranadacorresponde al traslado de animales a sectores con mejores recursos forrajeros. Respecto a la existencia de ganado trashumante, la Región de Coquimbo presenta 405.058 cabezas de ganado caprino, ocupando un porcentaje de 54.8\% (ODEPA 2014).

La búsqueda de nuevos pastizales y la difícil geografía de los campos precordilleranos han originado la adaptación de un tipo de raza de cabra: la cabra criolla, que es el resultado de cientos de años de crianza descontrolada y selección natural. Esta tiene su origen en razas españolas, traídas en tiempos de la conquista (Mujica 2005). Con el tiempo, personas de escasos recursos vieron en esta actividad un gran potencial para poder subsistir gracias a sus beneficios económicos. Sin embargo, el sobrepastoreo de la cabra criolla ha ocasionado la pérdida de la capa arbustiva que sostiene el suelo, dando paso a la erosión (Agraz 1981).

El objetivo del presente trabajo es conocer las plantas nativas utilizadas por los cabreros, con el fin de identificar sus usos y contribuir a establecer estrategias de conservación que permitan su continuidad en el tiempo.

\section{Materiales y métodos}

La quebrada de Tulahuén ( $30^{\circ} 57^{\prime} \mathrm{S}$ al $30^{\circ} 59^{\prime} \mathrm{S}$ y $70^{\circ} 42^{\prime} \mathrm{O}$ al $70^{\circ} 44^{\prime} \mathrm{O}$ ), Región de Coquimbo, es una extensión territorial que nace a partir de la zona montañosa del cerro de Tulahuén y baja hacia el lecho del río grande (Figura 1). En esta zona se ubican las majadas de cabreros que llevan a pastar a sus animales en la temporada de verano. Cada uno de ellos tiene su área de ocupación de esta actividad (Figura 1.)

En el área de estudio (Figura 2) se realizaron 10 parcelas de $50 \times 50$ m (Figura 4), con el fin de identificar las especies nativas y determinar la cobertura de las poblaciones allí presentes (Samo et.al. 2008; Steubing 2002). Los lugares donde se hicieron las parcelas fueron zonas cercanas por donde pasaba el camino de los cabreros, debido a la difícil geografía de la zona. La investigación se realizó entre diciembre y marzo del año 2014.

La primera parcela se ubica en una ladera de exposición con orientación Este, en la parte baja de Las Vegas; la segunda se sitúa en el Estero Villa; la tercera en el Estero de Los Lisos; la cuarta en el sector Quebrada Centro; la quinta en la ladera El Gigante; la sexta en una ladera de exposición Oeste; la séptima en la ladera de Los Lisos; la octava en la ladera de Los Acantilados; la novena en la ladera de La Pulpica y la décima en la quebrada Los Colorados. (Figura 4).

El reconocimiento de las especies nativas se realizó con la ayuda de la comunidad de cabreros, utilizando pequeños herbarios, más el conocimiento ancestral de las personas. Los nombres comunes de las especies existentes en las parcelas fueron identificados con la ayuda de un pequeño grupo de cabreros residentes en el lugar y consulta de material bibliográfico, como Rodríguez et al. 1983; Hoffmann et al. 1998; Ridemann \& Aldunate 2003; Ahumada \& Faúndez 2007; Ridemann et al. 2008; Teillier et al. 2011 y Hoffmann 2012.

En el trabajo con la comunidad de cabreros de la quebrada de Tulahuén se emplearon entrevistas semiestructuradas (Taylor \& Bogdam 1987), testimonios y fotografías, con el fin de conocer el uso de las plantas nativas e identificar los nombres comunes de las especies.

Con los estudiantes de la escuela se hicieron herbarios, para ayudar en la identificación de las especies. A las especies encontradas en este trabajo (Tabla III) se les asignó su origen (Zuloaga et al.2008) y su estado de conservación (MMA, consulta 2016; Squeo et al. 2001), según las categorías de la UICN. Hemos agregado una sigla de NE a todas aquellas especies que no han sido evaluadas bajo este criterio (Villaseñor \& Ramírez, 2016).

Una vez realizados los trabajos en terreno, se elaboró la cartografía del lugar identificando las majadas y los polígonos con la ayuda de los programas Google Earth y ArcMap10.1

Junto con esta metodología, las especies vegetales fueron determinadas en el Laboratorio 


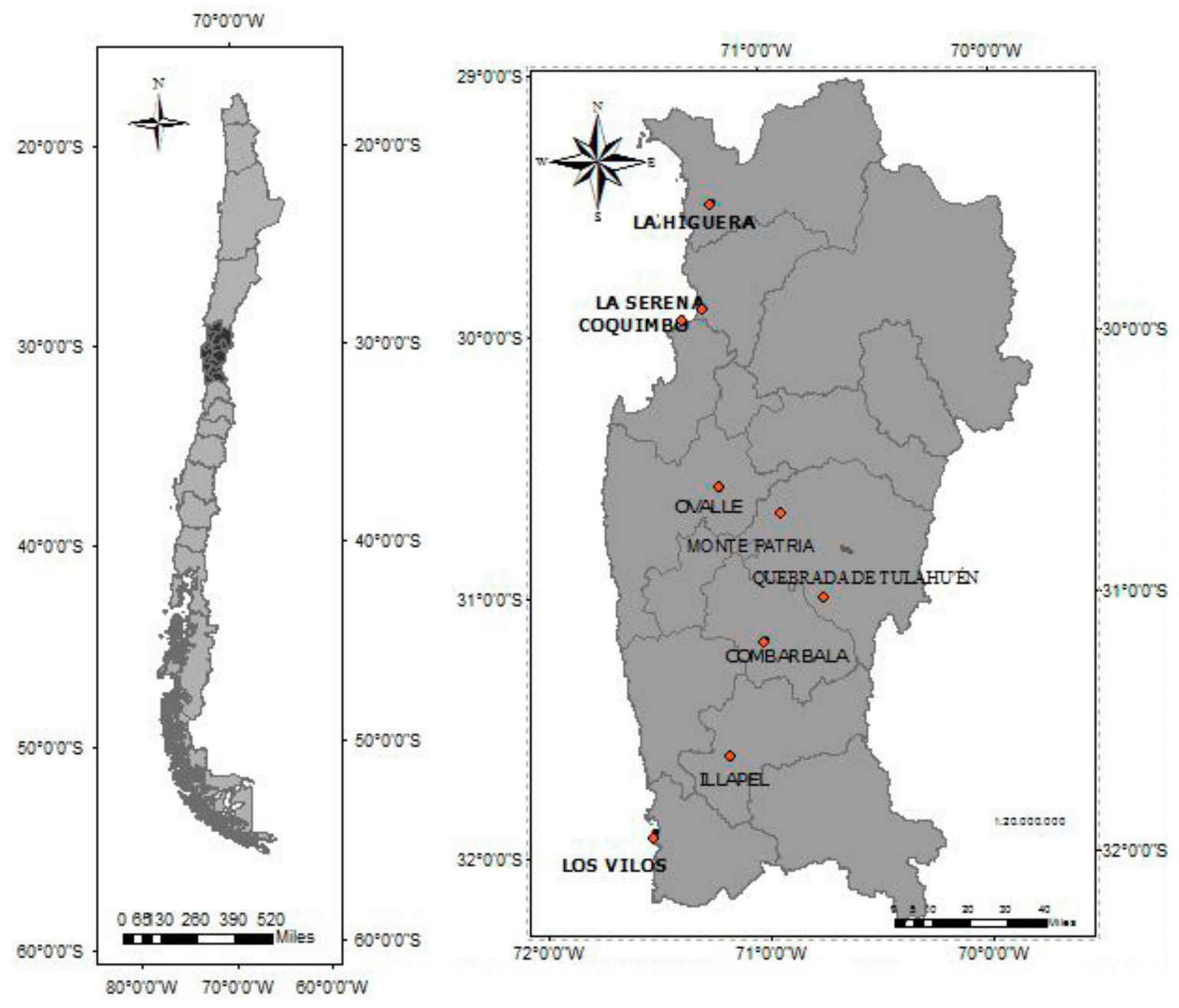

Figura 1. Cabreros Andinos Vegas de la Quebrada de Tulahuén Fuente: CEAZA, 2014. 


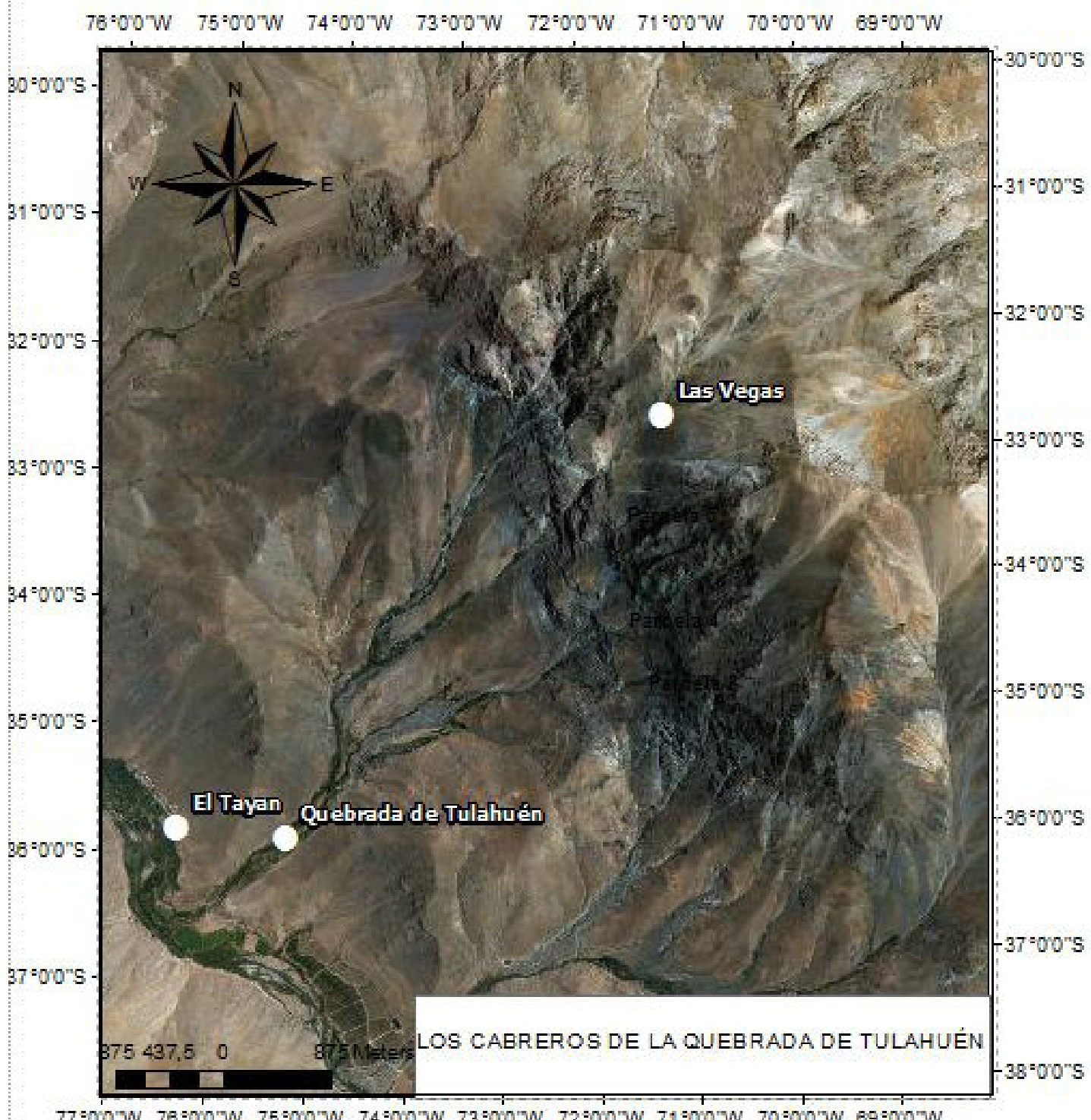

Figura 2. Zona de estudio. En la parte alta Las Vegas, la quebrada de Tulahuén y la localidad de El Tayan. 


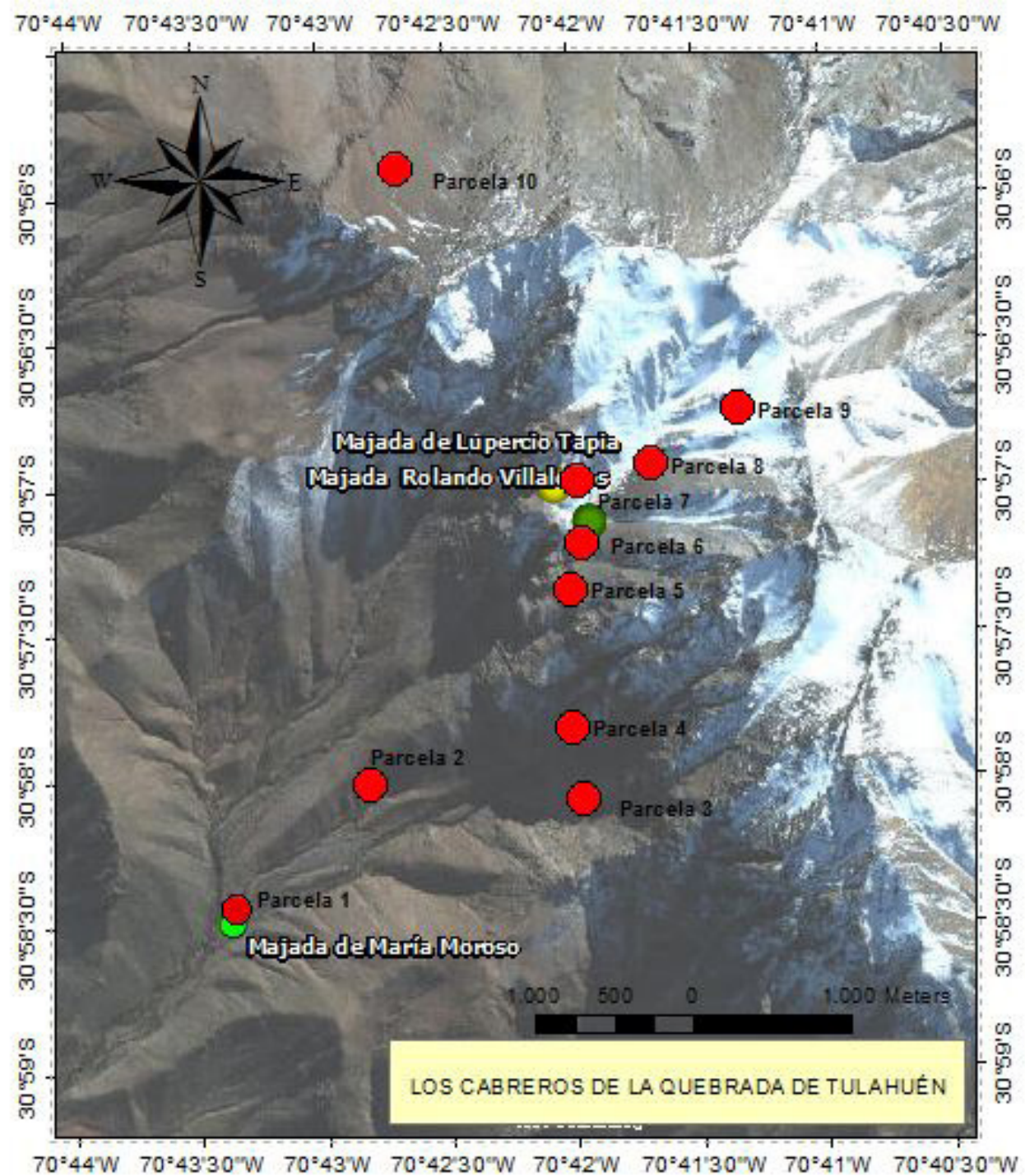

Figura 4. Sitio de estudio donde se ubican los cabreros andinos y las parcelas realizadas en los trabajos en terreno. El lugar es denominado por ellos como Las Vegas de la Quebrada de Tulahuén. Majadas de los cabreros que habitan en el sector precordillerano, de la provincia del Limarí. Fuente: CEAZA, 2014. 


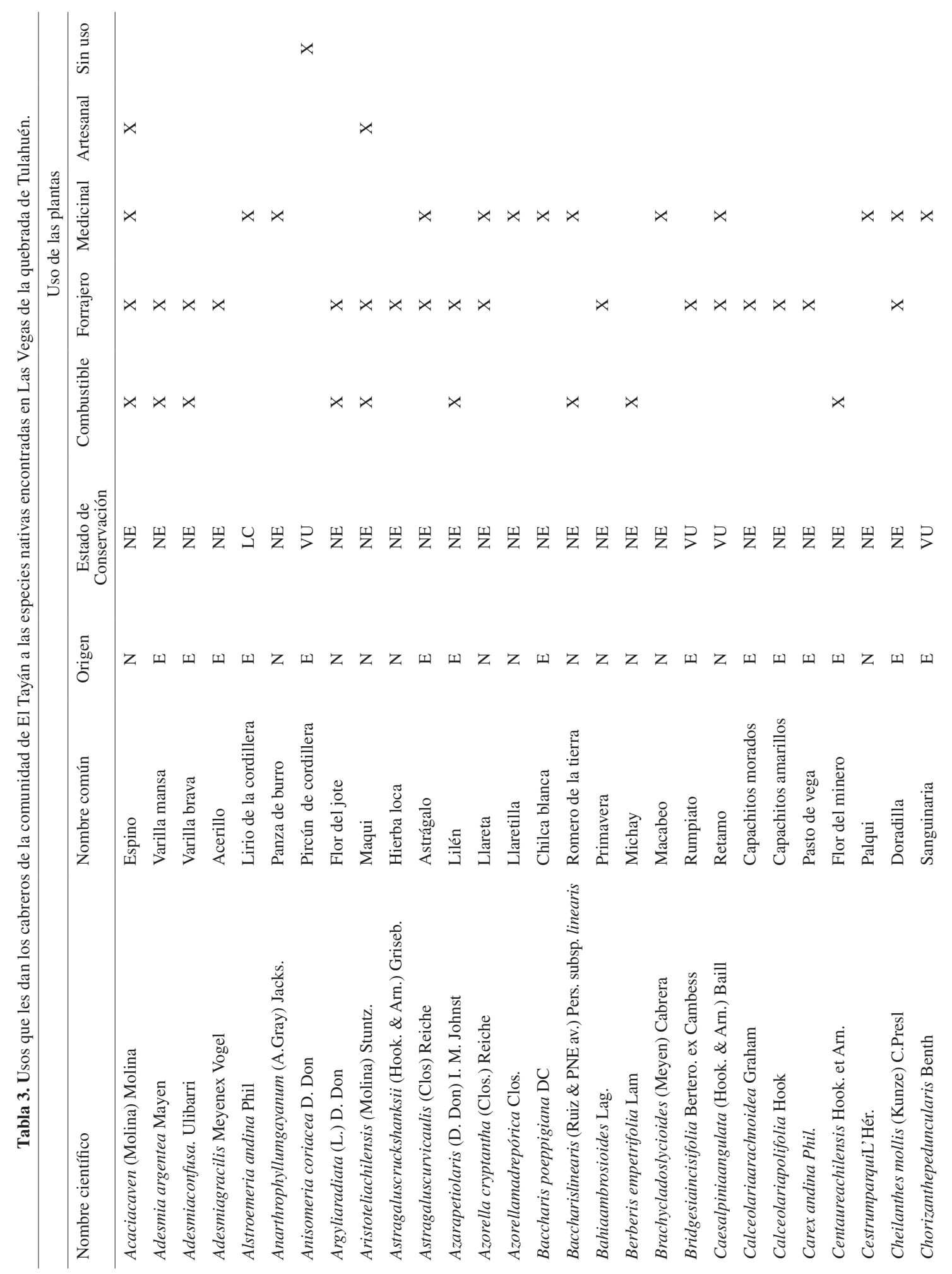




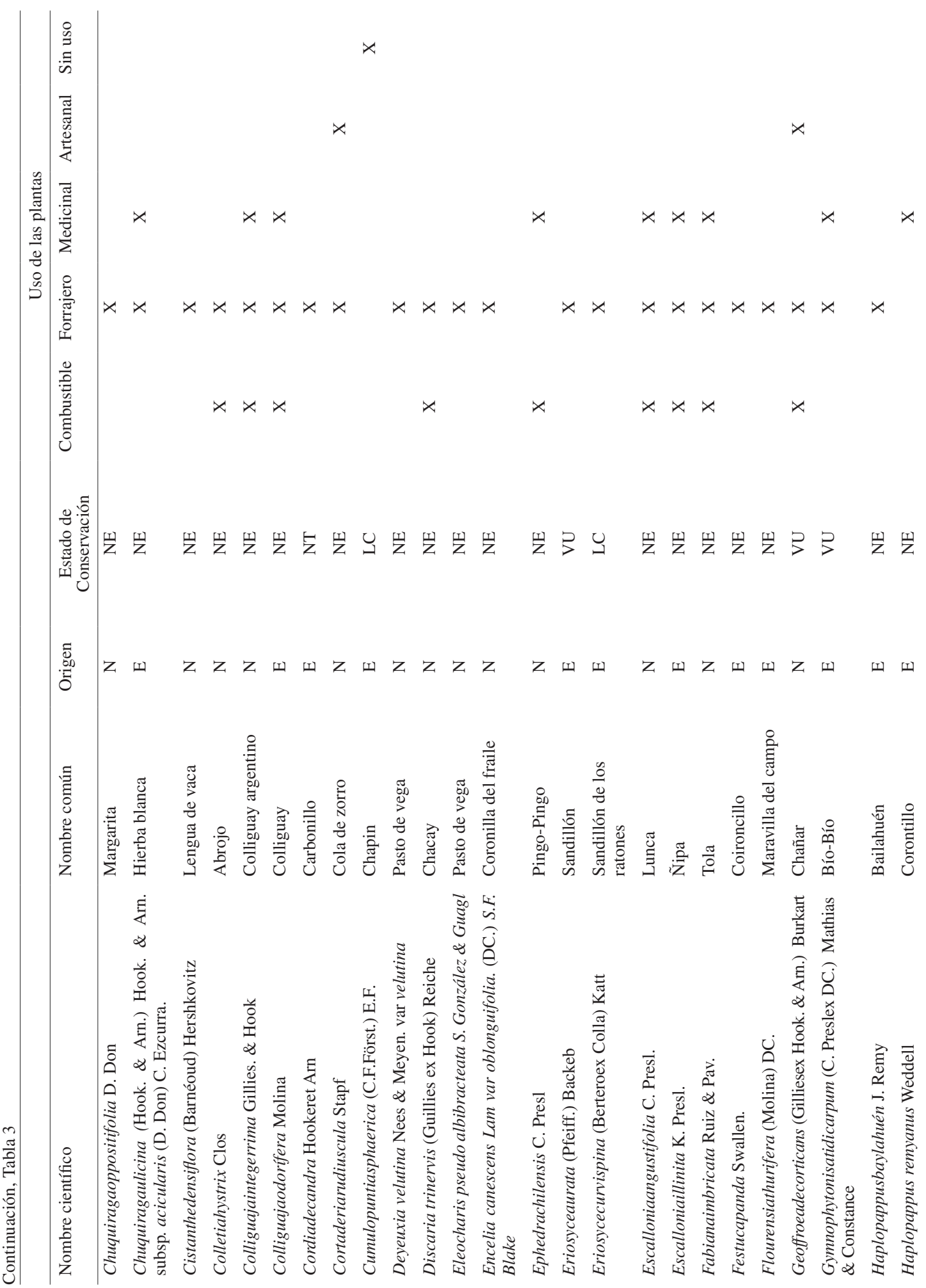




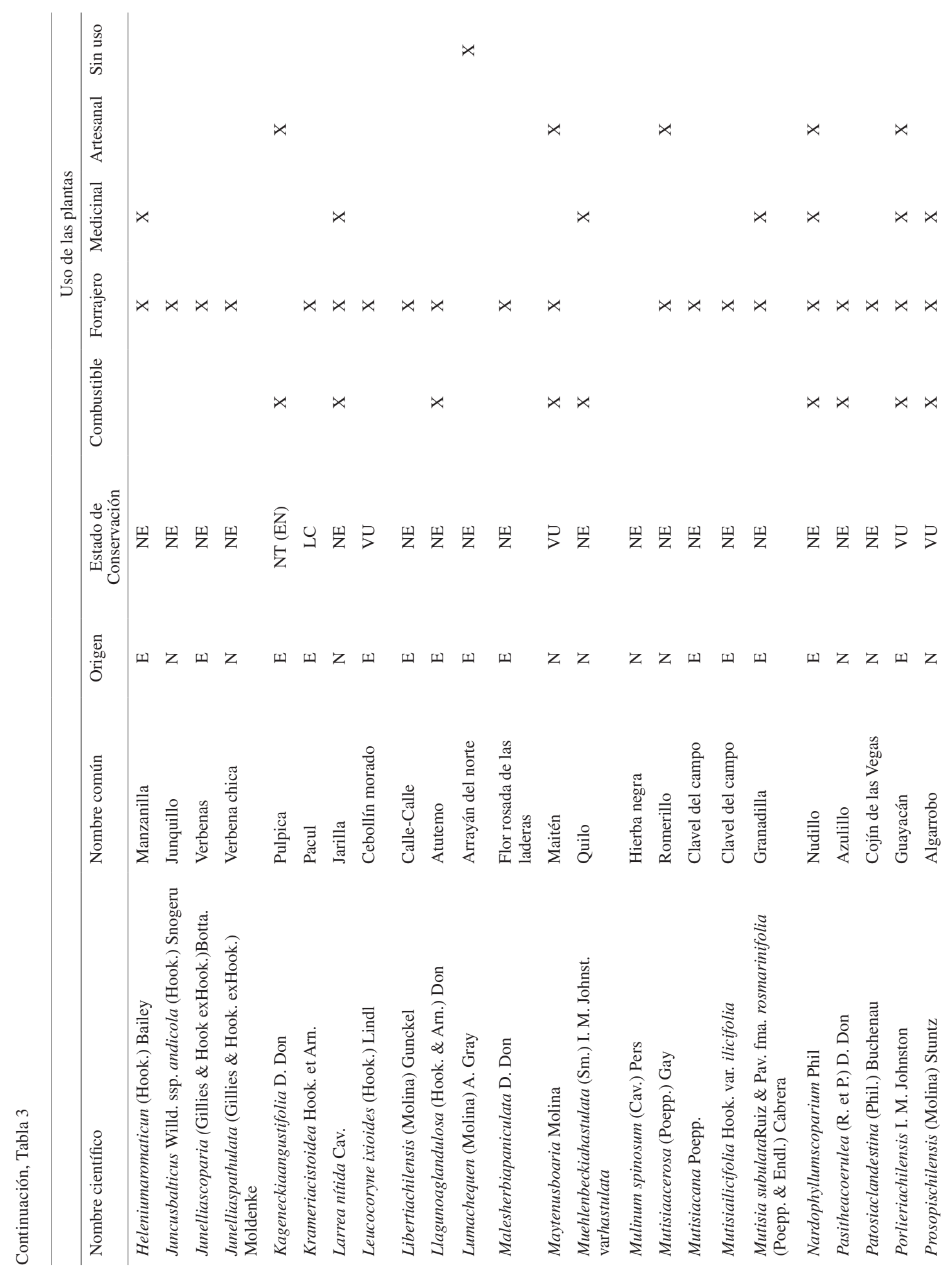




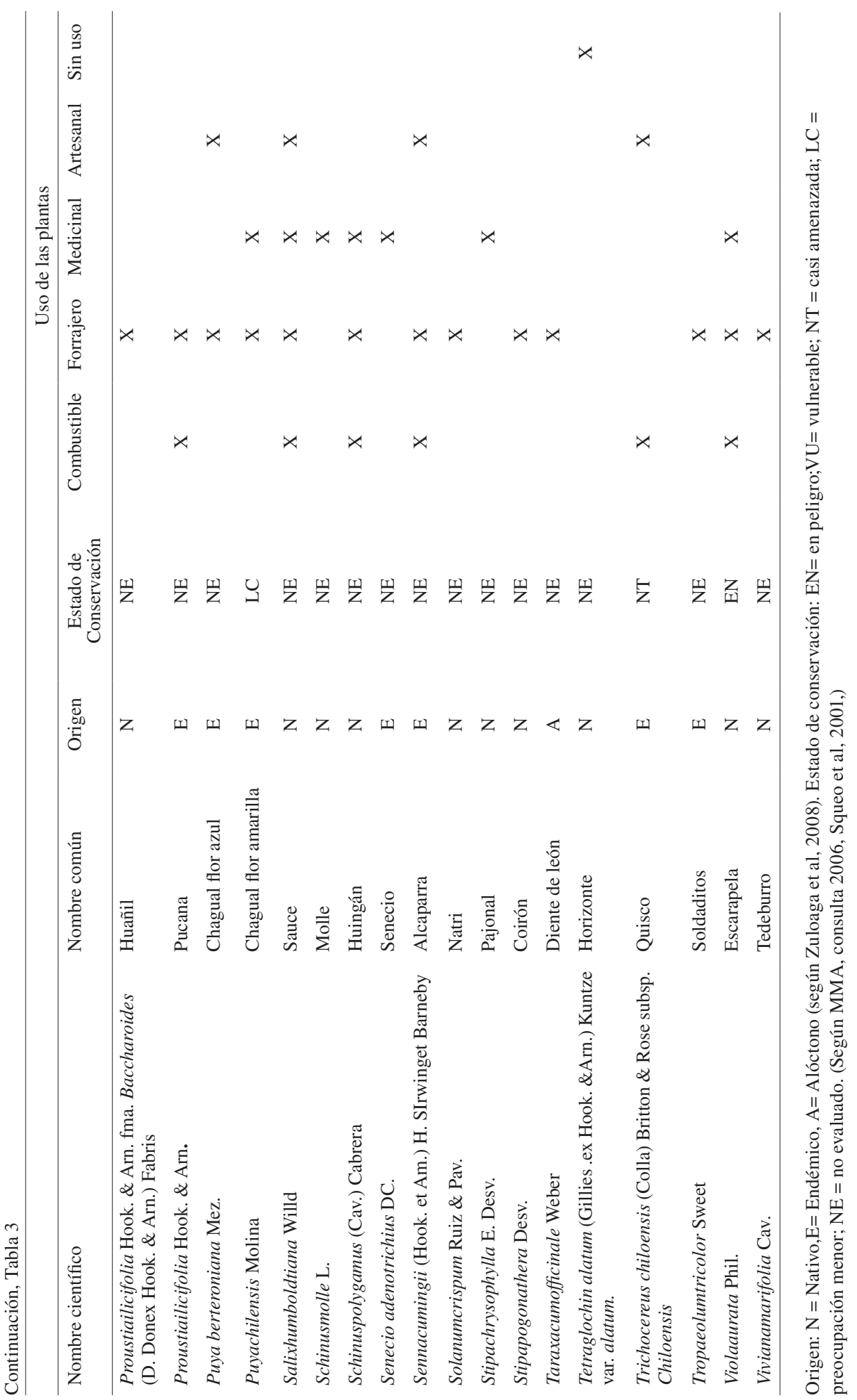


de Botánica de la Universidad de Playa Ancha, y depositadas en su herbario (VALPL).

\section{Resultados}

Las observaciones realizadas en terreno, especialmente en la parte alta de Las Vegas de la quebrada de Tulahuén, evidenciaron una escasa vegetación, asociada principalmente a arbustos de baja altura. Esta característica de la vegetación nativa y el pastoreo de las cabras han generado un aumento de suelos desnudos, donde es posible observar roca erosionada.

En la Figura 5 y la Tabla II, se indica la ubicación, los metros de altitud de cada parcela y la ubicación geográfica de ellas.

En la zona de estudio existe una vegetación donde se observan los siguientes pisos altitudinales: de 800 a 2.000 metros de altitud hay una fragmentación del bosque esclerófilo mediterráneo abierto, dominado por Kageneckia angustifolia D. Don (Pulpica) en la estrata arbórea y por Colliguaja integérrima Gillies \& Hook (Coliguay argentino) en la estrata arbustiva. Entre los $1.300 \mathrm{msnm}$ y los 2.600 se da una vegetación de quebrada, dominada por Maytenus boaria Molina (Maitén), Escallonia illinita K. Presl (Ñipa), Schinus polygamus Cav (Molle) (.) Cabrera) y Sauce (Salixhumboldtiana Willd). Entre los 800 y $2.500 \mathrm{msnm}$ se presenta, especialmente en laderas de solana, un matorral xerofítico con Colliguay (Colliguajaodorífera Molina), Pucana (Proustia ilicifolia Hook \& Arn.), Quisco (Trichocereuschiloensis (Colla) Britton \& Rose), Sandillón (Eriosyce aurata (Pfeiff.) Backeb.) Rumpiato (Bridgesia incisifolia Bertero ex Cambess) y Guayacán (Porlieria chilensis I. M. Johns).

De los 1.300 a los $3.200 \mathrm{msnm}$, en laderas de exposición S-O, especialmente, se presentan los bosques abiertos de tipo esclerófilo seco, en los que predominan la Pulpica, Lilén (Azara petiolaris (D. Don) I.M. Johnst.), Chacay (Discaria chacaye (G. Don) Tortosa), Jarrilla (Larrea nítida Cav.), Pacul (Krameria cistoidea Hook. \& Arn.).

Sobre los $3.000 \mathrm{msnm}$ hay arbustos de no más de 50 centímetros. Estos son, por lo general, achaparrados y retorcidos, como Margarita (Chuquiragaoppositifolia D. Don), Neneo (Milinum spinosum (Cav.) Pers), Michay (Berberis empetrifolia Lam.), apareciendo las primeras especies en cojines densos como la Llareta
(Azorella compacta Phil), que forman una estepa andina.

Las Vegas se ubican en la parte alta de la montaña del cerro de Tulahuén. Poseen una altura aproximada de $3.450 \mathrm{msnm}$. ILa formación vegetal que allí domina son los Pastos de vegas (Carex subantartica Speg),(Deyeuxia velutinaNees \& Meyen) y (Eleocharis lechleri Boek). Tienen una parte de sus empastadas con agua que escurre con la pendiente, conformando una quebrada que los lugareños conocen como quebrada de Tulahuén. (Figura 2); y también una estepa alto andina en la que dominan Coirón (Stipa pogonathera E, Desv.), Romerillo (Mutisia acerosa Poepp ex Less.) y Llaretilla (Azorella madrepórica Clos ex Gay).

En el sector de estudio de la quebrada de Tulahuén hay un total de 93 especies nativas y 1 asilvestrada (Tabla III). 49 de ellas son endémicas (51\%), 46 tienen representación al menos en Argentina (48\%) y una cosmopolita (1\%). De estas especies, 2 están en categoría de En Peligro (EN), 11 Vulnerables (VU), 2 Casi Amenazadas (NT) y 2 en Preocupación menor (LC), y las 73 restantes no han sido evaluadas bajo este criterio (NE).

De las especies que crecen en el sector, los cabreros y la gente de la comunidad de El Tayán, poblado cercano a la quebrada, reconocieron con nombres comunes a las 93 identificadas.

De las entrevistas semiestructuradas aplicadas a la comunidad de cabreros se obtuvieron los diferentes usos que les dan a las especies nativas. Para diferenciarlas se establecieron 5 parámetros de clasificación: combustible, artesanal, forrajera, medicinal y sin uso; y se asignaron 152 usos, considerando que algunas plantas tienen más de uno. (Tabla III).

32 especies son utilizadas como combustible, es decir, para cocinar los alimentos o hacer carbón. Destacan en esto la Pulpica, Maitén,Ñipa, Algarrobo (Prosopis chilensis (Molina) Stuntz) y Espino (Acacia caven (Molina). 72 plantas fueron citadas como forrajeras, y entre las más comunes se encuentran Rumpiato, Maravilla del campo (Flourensia thurifera Molina DC.) y Colliguay (Tabla III).

En el transcurso de esta investigación fue posible constatar un sentimiento de respeto y cercanía con el Rumpiato, ya que es una de las primeras plantas que brotan cuando caen 


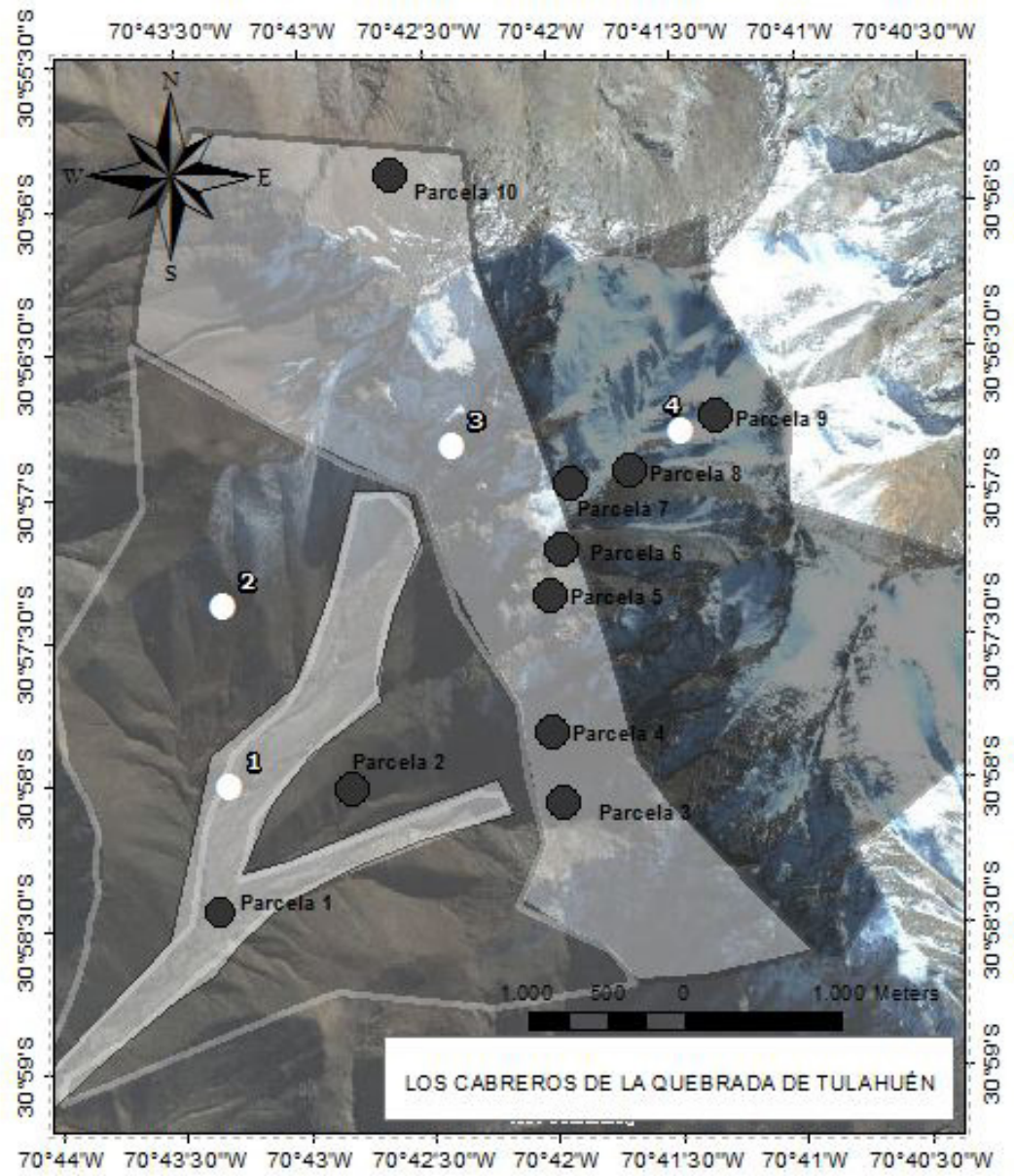

Figura 5. Formaciones vegetales reconocidas en el área de estudio. 1.- Bosque abierto húmedo; 2.- Matorral xerofítico; 3.- Bosque abierto seco; 4.- Estepa andina. Fuente: CEAZA, 2014.

Tabla 1. Resultados en entrevistas aplicadas a los cabreros de la quebrada de Tulahuén y al poblado El Tayán, comuna de Monte Patria, Región de Coquimbo.

\begin{tabular}{|c|c|c|c|c|}
\hline Entrevistados & $\begin{array}{l}\text { 1.- ¿Conoce } \\
\text { algún uso de las } \\
\text { plantas nativas? }\end{array}$ & $\begin{array}{l}\text { 2.-¿Quién le trasmitió el } \\
\text { conocimiento de los usos } \\
\text { de las plantas nativas? }\end{array}$ & $\begin{array}{l}\text { 3.- ¿Dónde van a buscar las } \\
\text { plantas nativas? }\end{array}$ & $\begin{array}{l}\text { 4.- Mencione la } \\
\text { especie nativa que } \\
\text { más recolectan }\end{array}$ \\
\hline Niños de 5 a 17 años & $\begin{array}{l}\mathrm{SI}=62 \% \\
\mathrm{NO}=38 \%\end{array}$ & $\begin{array}{l}\text { Abuelos }=49 \% \\
\text { Padres }=11 \% \\
\text { Parientes }=40 \%\end{array}$ & $\begin{array}{l}\text { Quebrada de Tulahuén }=31 \% \\
\text { Las Vegas }=29 \% \\
\text { Se desconoce }=40 \%\end{array}$ & $\begin{array}{l}\text { Romerillo }=31 \% \\
\text { Jarilla }=26 \% \\
\text { Pingo-pingo }=26 \% \\
\text { No menciona }=17 \%\end{array}$ \\
\hline Jóvenes de 18 a 30 años & $\begin{array}{l}\mathrm{SI}=51 \% \\
\mathrm{NO}=49 \%\end{array}$ & $\begin{array}{l}\text { Abuelos }=43 \% \\
\text { Padres }=9 \% \\
\text { Parientes }=48 \%\end{array}$ & $\begin{array}{l}\text { Quebrada de Tulahuén }=35 \% \\
\text { Las Vegas }=21 \% \\
\text { Se desconoce }=44 \% .\end{array}$ & $\begin{array}{l}\text { Jarrilla }=48 \% \\
\text { Calle-calle }=35 \% \\
\text { Romerillo }=17 \%\end{array}$ \\
\hline Adultos de 30 a 65 años & $\begin{array}{l}\mathrm{SI}=95 \% \\
\mathrm{NO}=5 \%\end{array}$ & $\begin{array}{l}\text { Abuelos }=85 \% \\
\text { Padres }=15 \%\end{array}$ & $\begin{array}{l}\text { Quebrada de Tulahuén }=62 \% \\
\text { Las Vegas }=33 \% \\
\text { Se desconoce }=5 \%\end{array}$ & $\begin{array}{l}\text { Llareta }=43 \% \\
\text { Bío-bío }=40 \% \\
\text { Calle-calle }=10 \% \\
\text { Palqui }=6 \% \\
\text { No menciona }=1 \%\end{array}$ \\
\hline Abuelos de más de 65 años & $\begin{array}{l}\mathrm{SI}=100 \% \\
\mathrm{NO}=0\end{array}$ & $\begin{array}{l}\text { Abuelos }=100 \% \\
\mathrm{NO}=0\end{array}$ & $\begin{array}{l}\text { Quebrada de Tulahuén }=50 \% \\
\text { Las Vegas }=50 \%\end{array}$ & $\begin{array}{l}\text { Sanguinaria }=40 \% \\
\text { Guayacán }(30 \%) \\
\text { Calle-calle }(30 \%)\end{array}$ \\
\hline
\end{tabular}




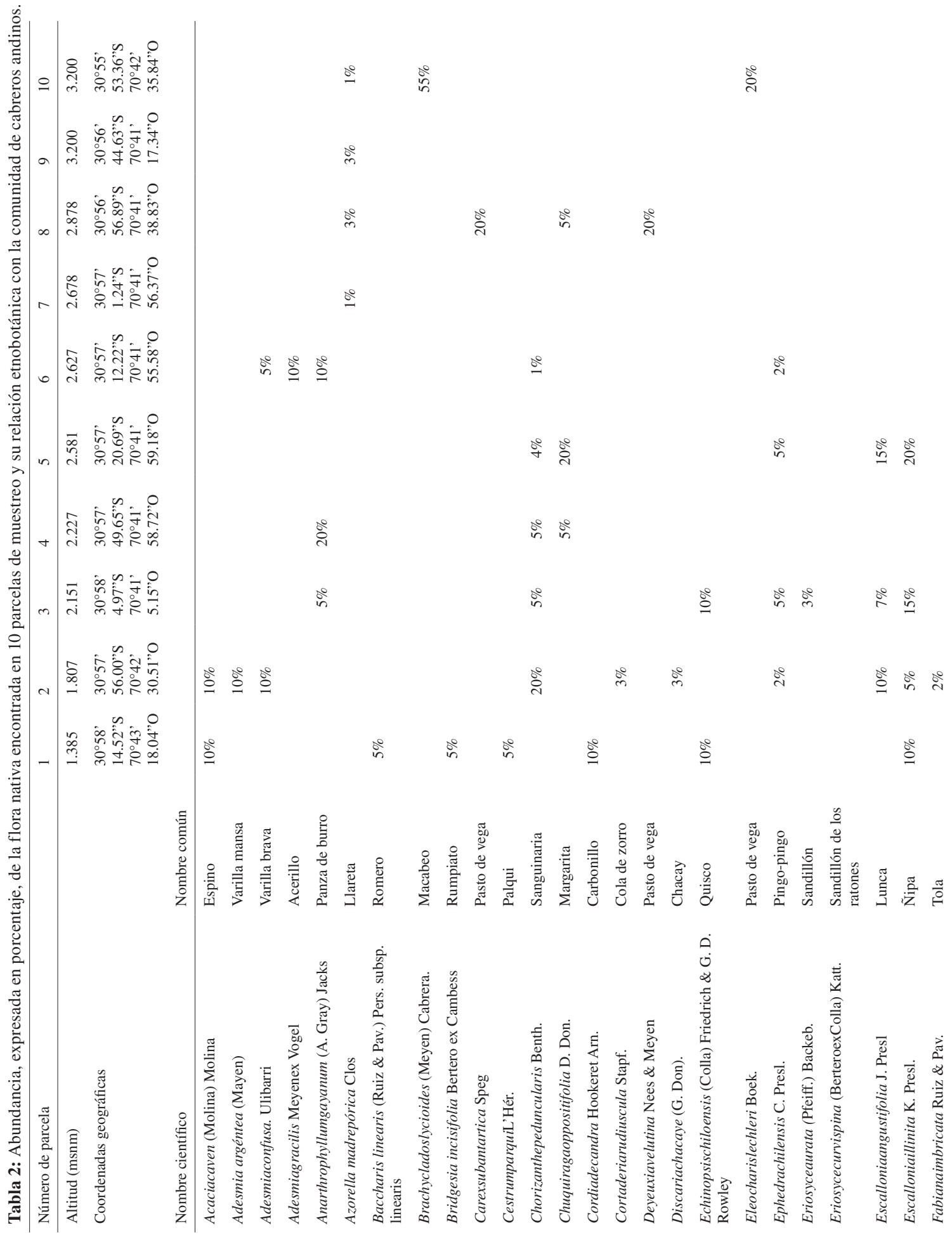




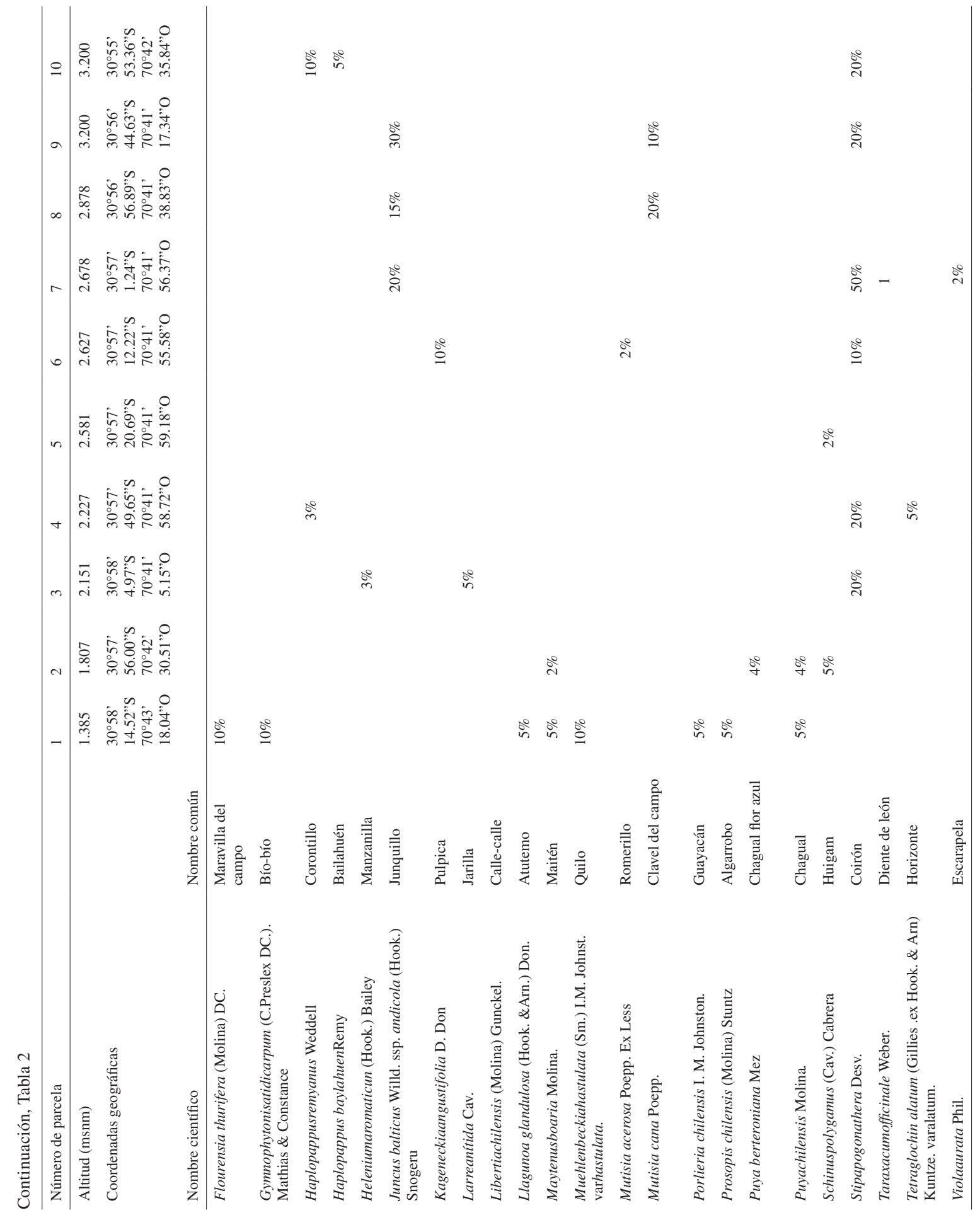


precipitaciones en el lugar, permitiendo alimentar a las cabras y así obtener ingresos económicos para las familias. Otros pastos muy consumidos por las cabras que crecen a gran altura son Coirión y Pastos de vegas.

35 especies fueron consideradas como medicinales, siendo muy llamativa la Llareta (Azorella madrepórica Clos), que es muy escasa en el sector, y se encuentra en los nacimientos de las vegas. El grupo de cabreros y pobladores de El Tayán siente una gran cercanía con la Llareta, pues afirman que es una planta escasa en el lugar y de gran ayuda. Toman aguas de esta hierba para aliviar algunas molestias urinarias y digestivas. Otras plantas medicinales son Romerillo y Macabeo (Brachyclados lycioides (D. Don) Cabrera). De estos vegetales los cabreros recolectan pequeñas ramas y hojas para llevarles a sus familiares en la ciudad. Ellos suelen decir que tomar aguas de estas plantas les ayuda a mantenerse sanos y regular la temperatura corporal.

En el lugar existen 13 especies utilizadas para fines artesanales (Tabla III). Según cuentan los cabreros es muy común que personas ajenas a la comunidad corten tallos y ramas de Guayacán y Pulpica, para tallar objetos como llaveros, lámparas y collares.

Sólo una planta fue considerada por los cabreros y las personas de la localidad de El Tayán sin uso: Chapín (Cumulopuntiasphaerica (C.F. Forst.) E.F). Esta es una Cactácea, considerada por muchos una mala planta debido a los dolores que causan sus espinas, que se pegan en el cuerpo cuando ellos sacan a pastar sus cabras.

Se entrevistó a un total de 160 personas: cabreros que habitan en la quebrada de Tulahuén, pobladores de El Tayán, asentamiento ubicado en las cercanías del área de estudio (3058'43.13"S y $\left.70^{\circ} 44^{\prime} 16.37^{\prime \prime} O\right)$, y a los niños de la escuela rural de esta localidad. En total se trabajó con 35 niños, 35 jóvenes, 80 adultos y 10 adultos mayores.

35 son niños de 5 a 17 años, estudiantes de la escuela; 35 jóvenes de 18 a 29 años, de los cuales 30 son temporeros y 5 cabreros; 80 adultos de 30 a 64 años, de los cuales 15 son dueñas de casa, 60 temporeros y 5 cabreros; y 10 "abuelos" de más de 65 años, de los cuales 8 son jubilados y dos cabreras.

En la entrevista se hicieron cuatro preguntas: 1.- ¿Conoce algún uso de las plantas nativas? 2.- ¿Quién le trasmitió el conocimiento de los usos de las plantas nativas? 3.- ¿Dónde van a buscar las plantas nativas? 4.- Mencione la especie nativa que más recolectan.

\section{Discusión}

Teillier (2011) define cuatro pisos de vegetación, desde los 1.800 a los $3.800 \mathrm{msnm}$, con siete formaciones vegetales, para la cordillera de los Andes de Santiago, Región Metropolitana. Estos pisos desde Subandino hasta Altoandino corresponden aproximadamente a los descritos en este trabajo, así como las formaciones vegetales. También la vegetación puede corresponder a los pisos de Luebert y Pliscoff (2006), "Matorral bajo desértico mediterráneo andino de Senecio proteus y Haplopappus baylahuen" y también al piso "Bosque esclerófilo mediterráneo andino de Kageneckia angustifolia y Guindilia trinervia", aunque sus composiciones florísticas no coinciden totalmente.

Para Las Vegas de bofedales y quebradas en el Loa Superior, Andes de Antofagasta, se determina que la edad y el lugar de residencia de los pobladores son factores significativos y que influyen en el grado de conocimiento de la flora estudiada (Villagrán \& Castro 1999). Esta tendencia también puede ser encontrada en los cabreros andinos que viven en la quebrada de Tulahuén. El grado de conocimiento de los usos de las plantas nativas es mayor en aquellos cabreros con edades de 65 años en adelante, según los datos de los entrevistados, en comparación con los niños. Ellos afirman que el $49 \%$ del conocimiento adquirido sobre el uso de la flora nativa viene de sus abuelos, el $11 \%$ de sus padres y el $40 \%$ lo han aprendido de parientes como sus hermanos, tíos, tías y primos que se dedican a la actividad caprina.

El grupo de los adultos entrevistados, cuyas edades van desde los 30 hasta los 65 años, mencionó que dentro de las plantas que más recolectan está el Palqui, con un $6 \%$ de las preferencias. Lo utilizan para barrer los hornos cuando hacen pan, curar el mal de ojo, entre otros usos. Sin embargo, consideran que es una mala hierba que producía la muerte a las cabras cuando estas se alimentaban de los brotes del vegetal. A diferencia del Norte de Argentina (cuenca del río Iruya, Salta), dondeCalifano \& Echazú (2013) registraron 11 especies vegetales tóxicas, 
en Las Vegas de la quebrada de Tulahuén sólo se encontró el Palqui.

En relación con el conocimiento de los usos de las plantas nativas en la comunidad de cabreros, los entrevistados mencionan que fue adquirido de sus antepasados. Esto coincide con el estudio de Toledo \& Kutschker (2012), donde el 93,75\%de los entrevistados de la Patagonia argentina manifestaron haber adquirido conocimiento sobre el uso de las plantas a través de sus familias, y se fue transmitiendo de manera informal.

Lo preocupante de los cabreros de Tulahuén es que han manifestado que cada día son menos las personas que se dedican al rubro. En el área de estudio figuran 10 adultos mayores, de los cuales 3 son hombres y 7 mujeres, y solo dos se dedican a la actividad caprina. Todos han asegurado conocer los usos de las plantas nativas. Esta situación dificulta el traspaso de nuevos conocimientos a los más jóvenes, ya que son los crianceros adultos los que han tratado de divulgar el uso de las plantas nativas, para poder vivir en estas zonas extremas.

En relación con las plantas medicinales encontradas dentro de la comunidad de cabreros, estos reconocieron que cada día es más difícil recolectar ramas de esos vegetales, porque deben caminar grandes distancias para ir a buscarlas y exponerse al peligro de accidentes.

Las bajas coberturas estimadas en las parcelas muestrales (Tabla II) indican que las condiciones ecológicas son bastante desfavorables para el desarrollo de la vegetación. A esto se agrega el sobrepastoreo que se ha producido por la gran cantidad de cabras que pastan allí (más de 2.000) (Figura3).El problema se agrava si consideramos que en la lista de especies encontradas aparecen varias

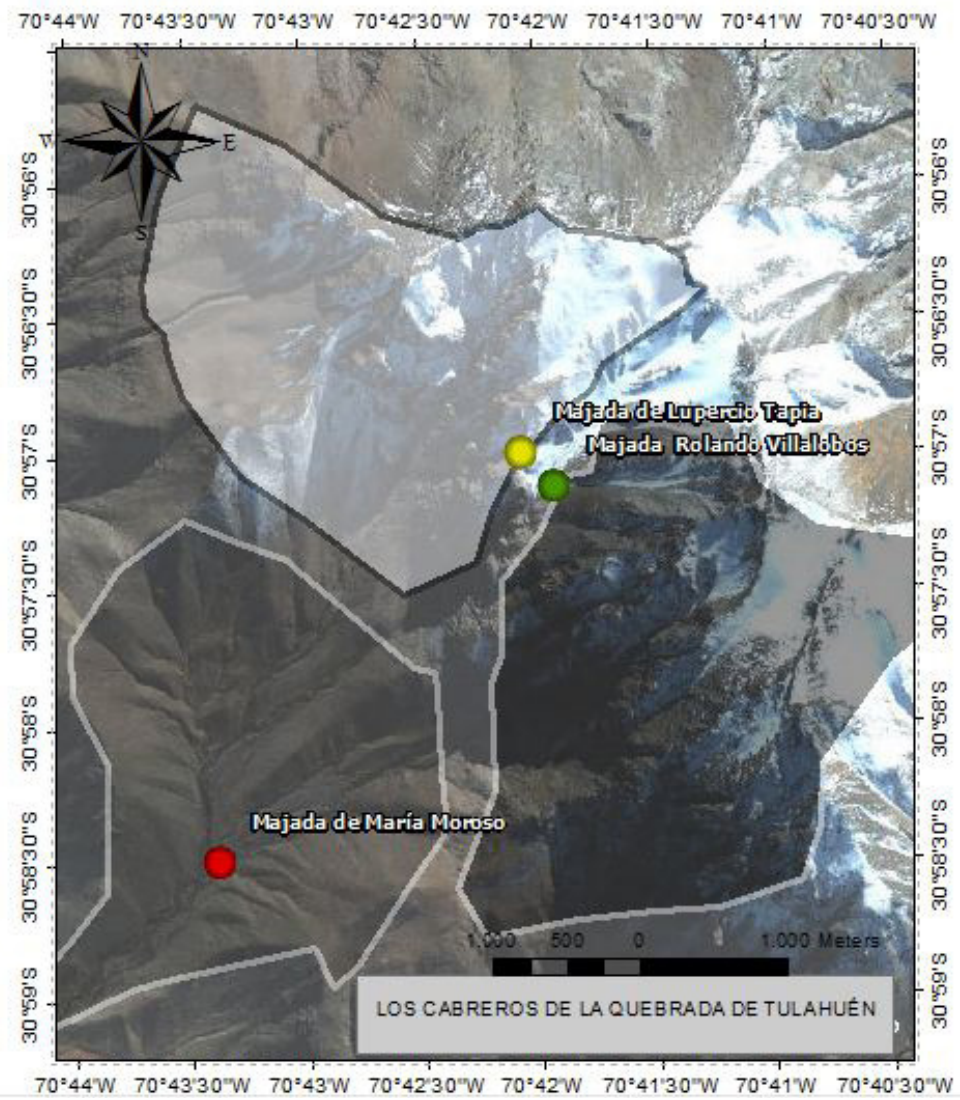

Figura 3. En el mapa. En la parte baja la majada de María Moroso, con 800 cabras ocupando una superficie aproximada de 10.56 kilómetros. En algunas ocasiones se juntan con las cabras de los otros ganados. En la parte alta la majada de Rolando Villalobos con 1.000 cabras, ocupando una superficie aproximada de 12,65 kilómetros dentro del área de estudio; y la majada de Lupercio Tapia con 300 cabras ocupando una superficie 17, 28 kilómetros. 
que están en alguna categoría de conservación. Por ejemplo: Kageneckia angustifolia (Squeo et al. 2001) y Viola aurata (MMA, consulta 2016) están en categoría En Peligro (EN); Anisomeria coriácea, Bridgesia incisifolia, Caesalpinia angulata, Chorizanthe peduncularis, Eriosyce aurata, Geoffroea decorticans, Gymnophyton isatidicarpum, Porlieria chilensis y Prosopis chilensis en categoría de Vulnerables (MMA, consulta 2016).
Según Squeo et al. 2001, se estableció el estado de conservación (categorías EX= Extinta, EP= En Peligro, VU: Vulnerable y FP= Fuera de Peligro) al $84,7 \%$ de las especies conocidas para cada comuna. La comuna de Monte Patria se sitúa dentro de los grupos intermedios en su proporción de la flora en las categorías EP=En Peligro o VU=Vulnerable, con un $(10,7 \%)$, antecedente que debería ser recogido en futuras acciones de conservación donde podrían hacer trabajo en conjunto con los cabreros.

\section{Literatura citada}

Agraz, A.

1981. Cría y explotación de la cabra en América Latina Editorial Hemisferio Sur. S.A. 481p.

Califano, L.; Echazú, F.

2013. Etnobotánica en comunidades pastoriles. Conocimiento tradicional sobre especies tóxicas para el ganado en La Cuenca del río Iruya (Salta Argentina). Boletín de la Sociedad Biológica Argentina. Botanica. 48 (2): 365-375.

Gajardo, R.

1994.La vegetación natural de Chile. Clasificación y distribución geográfica. Editorial Universitaria, CONAF, Santiago. 165 pp.

Hoffmann, A.

2012.Flora silvestre de Chile. Zona Central. Quinta edición. Ediciones Fundación Claudio Gay. Santiago, Chile. 254 pp.

Hoffmann, A.; Kalin, M.; Liberona, F.; Muñoz, M.; Watson, Y.J. 1998.Plantas alto andinas en la flora silvestre de Chile. Ediciones Fundación Claudio Gay. Santiago, Chile, 281 p.

Luebert, F.; Pliscoff, P.

2006. Sinopsis bioclimática y vegetacional de Chile. Editorial Universitaria. Santiago, Chile. 316 p.

Mujica, F.

2005. Razas caprinas, en el Instituto de Investigaciones Agropecuarias, Osorno, Chile. Instituto de Investigaciones Agropecuarias. Boletín INIA N²127. 88 p.

ODEPA.

2014. Ministerio de Agricultura. Informe Regional. Coquimbo, Chile. 15 p.

Ridemann, P.; Aldunate, G.

2003. Chile Zona Central. Editorial Andrés Bello. Santiago. 566 p.

Ridemann,P.; G. Aldunate, G; Teillier, S.

2008. Flora nativa de valor ornamental, identificación y propagación. Chile Zona Cordillera de los Andes. Editorial Andrés Bello. Santiago. 674 p.

Rodríguez, R.; Matthei, O.; Quezada. M.

1983. Flora arbórea de Chile. Editorial de la Universidad de Concepción. Concepción, Chile. 408 p.
Samo, A.; Garmendia, A.; Delgado, J.

2008. Introducción a la práctica de la ecología. PearsonPrentice Hall. Madrid, España. 175 p.

Squeo F.: Arancio, G.; Gutiérrez, J.

2001. Libro Rojo de la Flora Nativa y los Sitios prioritarios para su conservación: Región de Coquimbo, Chile. Ediciones Universidad de La Serena. 372 p

Steubing, L., Godoy, R.; Alberdi, M. 2002. Métodos de ecología vegetal. Monografías. Universidad Austral de Chile. Valdivia Chile. 345 p.

Taylor, S. J.; Bogdan, R.

1987. Introducción a los métodos cualitativos en investigación, la búsqueda de significados. Editorial PAIDOS. Barcelona, España. 174 p.

Teillier, S.; Marticorena, S.: Niemeyer, H.

2011. Flora Andina de Santiago. Guía para la identificación de las especies de las cuencas del Maipo y del Mapocho. Editorial Universidad de Chile. Santiago. Chile. 478 p.

Toledo, C., Kutschker, A.

2012. Plantas medicinales en el Parque Nacional Los Alerces, Chubut, Patagonia Argentina. Revista científica del Boletín la Sociedad Biológica Argentina. Bot. 47. (3-4): 461-470.

Villagrán C.; Castro, V.

1999. Etnobotánica y manejo ganadero de las Vegas, bofedales y quebradas en el Loa superior, Andes de Antofagasta, Segunda Región, Chile. Arica, Chile. Revista Chungara, 29 (2): 275-304.

Villaseñor, R.; Ramírez, P.

2016. Guía para el reconocimiento de especies del Santuario de la Naturaleza Acantilados Federico Santa María. Universidad de Playa Ancha. Valparaíso. Chile. 120 p.

Zuloaga, F.O.; Morrone, O.; Belgrano. M.J.

2008. Catálogo de las Plantas Vasculares del Cono Sur (Argentina, Sur de Brasil, Chile, Paraguay y Uruguay). Monographs of the Missouri Botanical Garden Tres volúmenes. $3.348 \mathrm{p}$. 